\title{
A NEW MODIFIED GOODNESS OF FIT TESTS FOR TYPE 2 CENSORED SAMPLE FROM NORMAL POPULATION
}

\author{
Ahmed M. Sultan \\ Egyptian Air Force \\ Cairo, Egypt \\ amsultan52@hotmail.com
}

\author{
Hala Mahmoud Khaleel \\ Zagazig University \\ Zagazig, Egypt \\ halamahmoud@hotmail.com
}

\begin{abstract}
In this paper a procedure is considered for a new modified goodness of fit test for the normal type 2 censored population. Samples of sizes 10(10)60 are chosen and censored at a specified percentage. Cramer von Mises and Anderson Darling test statistics are used with a nonparametric density estimator in place of the empirical distribution function. Tables of critical values for the two tests are generated. The power of the tests for nine alternative distributions is shown. Results show high power of discriminating the alternatives.
\end{abstract}

Keywords- Goodness of fit, Nonparametric estimation, Monte Carlo technique.

\section{INTRODUCTION}

An important problem in statistics is to find information about the form of the population from which a sample is drawn. Goodness of fit tests are given for the normal distribution with unknown mean and unknown variance from type 2 censored samples. We use the modified Cramer-von Mises (CvM) and Anderson-Darling (AD) goodness of fit tests.

A Monte Carlo procedure is used to develop and compare the modified goodness of fit (GOF) tests from censored samples. Critical values for different sample sizes $\mathrm{n}$ are generated. Let $r_{1}$ be the percentage at which a sample will be censored. In order to find which order statistic will determine the largest observation in the sample, let us define $r$ as an integer that rounds $r_{1} \times n$ to the largest integer not greater than $r_{1} \times n$. Thus, if $r_{1}=60 \%$, for a sample of size 20 , the sample will be censored at the $12^{\text {th }}$ order statistic. Samples of sizes $10(10) 60$ (i.e. sample sizes started at $n=10$ and ends at $\mathrm{n}=60$ with a step of 1 ) are chosen and censored at the $r^{\text {th }}$ order statistic. The modified CvM and AD test statistics are calculated for the given values of $\mathrm{n}$. This procedure is repeated 10000 times for each test statistic. These 10000 values are then ranked, and we find the $80 \%, 85 \%, 90 \%, 95 \%$, and $99 \%$ quantiles. These quantiles approximate the critical values for respective significance levels of $0.20,0.15,0.10$, 0.05 , and 0.01 for each test. Tables of critical values for the two modified test statistics from type 2 censored samples for the normal model are found. Also the power study of the modified tests to compare the efficiency of the $\mathrm{CvM}$ and $\mathrm{AD}$ tests for different sample sizes is discussed.

\section{THE NORMAL DISTRIBUTION}

The normal distribution is without a doubt the most important and most widely used continuous probability distribution. It is the fundamental base of the application of statistical inference in analysis of data, because the distributions of several important sample statistics tend toward a normal distribution as the sample sizes increase. 
The probability density function of the normal distribution is given by

$$
\begin{aligned}
& f(x)=\frac{1}{\sigma \sqrt{2 \pi}} e^{-(x-\mu)^{2} / 2 \sigma^{2},}, \\
& -\infty<x<\infty,-\infty<\mu<\infty, \sigma>0,
\end{aligned}
$$

where the parameters $\mu$ and $\sigma^{2}$ are the mean and variance, respectively.

Let $x_{(1)}, x_{(2)}, \ldots, x_{(n)}$ represent an order sample from a normal population. If some of these observations are not observed, the sample is said to be censored. At the $r^{\text {th }}$ order statistic, if all the observations less than $x_{(r)}$ are missing, then the sample is known as left-censored, and if all the observations greater than $x_{(r)}$ are missing, it is right-censored or type 2 censoring. In this paper we consider the case of type 2 censoring.

Maximum likelihood estimates are complicated to calculate and percentage points of the test statistics for finite $n$ appear to converge more slowly to the asymptotic points when these estimates are used (D'Agostino and Stephens 1986). Gupta (1952) suggested estimates of $\mu$ and $\sigma$ which we used here, these are linear combinations of the available order statistics

$$
\begin{gathered}
\mu^{*}=\sum_{i=1}^{r} b_{i} x_{(i)}, \text { and } \sigma^{*}=\sum_{i=1}^{r} c_{i} x_{(i)} \\
\text { where } b_{i}=\frac{1}{r}-\frac{\bar{m}\left(m_{i}-\bar{m}\right)}{\sum_{i=1}^{r}\left(m_{i}-\bar{m}\right)^{2}}, \quad c_{i}=\frac{m_{i}-\bar{m}}{\sum_{i=1}^{r}\left(m_{i}-\bar{m}\right)^{2}}
\end{gathered}
$$

and $m_{i}$ is the expected value of the $i^{t h}$ order statistic of a sample of size $\mathrm{n}$ from the standard normal distribution and where $\bar{m}=\sum_{i=1}^{r} \frac{m_{i}}{r}$. Values of $m_{i}$ are tabulated or can be well approximated by Blom(1958):

$$
m_{i}=\Phi^{-1}\left(\frac{i-0.375}{n-0.125}\right)
$$

where $\Phi^{-1}$ (.) is the inverse C.D.F of the standard normal, and the estimates $\mu^{*}, \sigma^{*}$ have been shown to be asymptotically efficient (Ali and Chan 1964). These estimates are the same as those obtained by least squares when $x_{i}$ is regressed against $m_{i}, i=1, \ldots, r$ (D'Agostino and Stephens 1986).

\section{GOODNESS OF FIT TEST STATISTICS FOR TYPE 2 CENSORED DATA}

Goodness of fit tests (GOF) measure the degree of agreement between the distribution of an observed data sample and the theoretical statistical distribution. 
A goodness of fit test based on the empirical distribution function (EDF), where the parameters are estimated, is called a modified goodness of fit test.

EDF statistics are based on the vertical differences between the empirical distribution function $F_{n}(x)$ and the theoretical statistical distribution $F(x)$ and they are divided into two classes, the supremum class and the quadratic class.

\section{The supremum statistics class:}

This includes, $D^{+}$and $D^{-}$defined as:

$$
D^{+}=\sup _{x}\left\{F_{n}(x)-F(x)\right\} \text {, and } D^{-}=\sup _{x}\left\{F(x)-F_{n}(x)\right\} \text {. }
$$

the most well known EDF statistic is $D$

$$
D=\sup _{x}\left|F_{n}(x)-F(x)\right|=\max \left(D^{+}, D^{-}\right) \text {. }
$$

The quadratic statistics class:

A second and wide class of measures of discrepancy is given by the Cramervon Mises family

$$
Q=n \int_{-\infty}^{\infty}\left\{F_{n}(x)-F(x)\right\}^{2} \Psi(x) d F(x)
$$

where $\Psi(x)$ is a suitable function, which gives weights to the squared difference $\left\{F_{n}(x)-F(x)\right\}^{2}$. When $\Psi(x)=1$ the statistic is the Cramer-von Mises statistic CvM denoted by $W^{2}$, and when $\Psi(x)=[\{F(x)\}\{(1-F(x))\}]^{-1}$ the statistic is the Anderson-Darling

We will define the goodness of fit test statistics for type 2 censored data. First, for a specified sample of size $\mathrm{n}$ from the normal population, we get the ordered type 2 censored statistics $x_{(1)}<x_{(2)}<\ldots<x_{(r)}$ and suppose the distribution of $x$ is $F(x)$. Based on this sample the probability integral transformation defined by:

$$
z_{(i)}=F\left(x_{(i)}\right)
$$

is itself censored, i.e. $z_{(1)}<z_{(2)}<\ldots<z_{(r)}$ with $z_{(r)}$ corresponding to the largest observation.

Pettitt and Stephens (1976) introduced versions of the Cramer-von Mises ${ }_{2} W_{r, n}^{2}$, and Anderson-Darling $2 A_{r, n}^{2}$ statistics, obtained for type 2 censored data by modifying the upper limit of integration in the definition of these statistics, given $z_{(1)}<z_{(2)}, \ldots<z_{(r)}$ the formulas are

$$
\begin{aligned}
{ }_{2} W_{r, n}^{2}= & \sum_{i=1}^{r}\left(z_{(i)}-\frac{2 i-1}{2 n}\right)^{2}+\frac{r}{12 n^{2}}+\frac{n}{3}\left(z_{(r)}-\frac{r}{n}\right)^{3}, \\
{ }_{2} A_{r, n}^{2}= & \left.-\frac{1}{n} \sum_{i=1}^{r}(2 i-1)\left[\log z_{(i)}-\log 1-z_{(i)}\right\}\right]-2 \sum_{i=1}^{r} \log \left(-z_{(i)}\right\} \\
& \left.-\frac{1}{n}\left[(r-1)^{2} \log 1-z_{(i)}\right\}-r^{2} \log _{(r)}+n^{2} z_{(r)}\right]
\end{aligned}
$$


To this point, an approach depending on the replacement of the EDF by the nonparametric density is used. The kernel estimator with Gaussian kernel is defined by:

$$
\begin{aligned}
\hat{f}(x) & =\frac{1}{n h} \sum_{i=1}^{n} k\left(\frac{x-x_{i}}{h}\right) \\
& =\frac{1}{n h} \sum_{i=1}^{n} \frac{1}{\sqrt{2 \pi}} \exp -\frac{1}{2}\left(\frac{x-x_{i}}{h}\right)^{2}
\end{aligned}
$$

is given as: $\hat{F}(x)$ The distribution function C.D.F. of this kernel density

$$
\begin{aligned}
\hat{F}(x) & =\int_{-\infty}^{x} \frac{1}{n h} \sum_{i=1}^{n} \frac{1}{\sqrt{2 \pi}} \exp -\frac{1}{2}\left(\frac{x-x_{i}}{h}\right)^{2} d x \\
& =\frac{1}{n} \sum_{i=1}^{n} \int_{-\infty}^{x} \frac{1}{\sqrt{2 \pi} h} \exp -\frac{1}{2}\left(\frac{x-x_{i}}{h}\right)^{2} d x \\
& =\frac{1}{n} \sum_{i=1}^{n} \Phi\left(\frac{x-x_{i}}{h}\right)
\end{aligned}
$$

where $\Phi(x)$ denotes the C.D.F. for the standard normal random variable, and the kernel function $K$ is a symmetric probability density function on the entire real line.

In equations (3-3) and (3-4) the expression of the EDF will be replaced by $\hat{F}(x)$, the cumulative distribution function C.D.F of the nonparametric kernel estimator $\hat{f}(x)$, for $\mathrm{i}=1,2, . ., \mathrm{r}$, and the bandwidth $\mathrm{h}$ will be taken $\mathrm{h}=1.06 \mathrm{Sn}^{-1 / 5}$ (Silverman 1986), where $\mathrm{S}$ is the sample standard deviation.

\section{CRITICAL VALUES}

Critical values for the modified goodness of fit tests are generated using Monte Carlo procedure. Lilliefors (1967) first used this approach to find tables of critical values for a modified Kolmogrov-Smirnov (K-S) test for the normal distribution with estimated mean and variance. And for the exponential distribution with unknown mean, Lilliefors (1967) were introduced with a study of the power of the test, which showed that the modified K-S test had higher power than $\chi^{2}$-test for the normal case.

Choulakian, Lockhart and Stephens( 1994) developed the Cramer-von Mises statistics for use in testing the discrete distribution and gave tables for tests for the discrete uniform distribution.

Now suppose the sample is type 2 censored, and the null hypothesis is $H_{0}$ : the censored sample $x_{(1)}<x_{(2)} \ldots<x_{(r)}$ comes from the normal distribution $F(x)$, with unknown mean and unknown variance.

The Monte Carlo procedure for the critical values determination can be described in the following 9 steps for the null hypothesis: 
Step 1 A sample of $\mathrm{n}$ normal random variates $x_{1}, x_{2}, \ldots, x_{n}$ is generated from the normal distribution with mean 100 and variance 10, n takes the values 10(10) 60 using the RNNOR routine from IMSL library.

Step 2 The random variates are converted to order statistics by sorting them in ascending order, then the ordered sample is censored at the $r^{\text {th }}$ order statistic as defined in section 1 .

$\underline{\text { Step } 3}$ The normal parameters $\mu^{*}$ and $\sigma^{*}$ are obtained using equation (2-2).

Step 4 Find $w_{i}=\left\{x_{(i)}-\mu^{*}\right\} / \sigma^{*}, i=1,2, \ldots r$.

$\underline{\text { Step } 5}$ Calculate $z_{(i)}=\Phi\left(w_{i}\right)$ where $\Phi($.$) is the standard normal C.D.F.$

$\underline{\text { Step } 6}$ Find a continuous nonparametric estimator $\hat{F}(x)$ as in equation (3-6) for $\mathrm{i}=1,2 \ldots, \mathrm{r}$.

$\underline{\text { Step } 7}$ The modified ${ }_{2} W_{r, n}^{2},{ }_{2} A_{r, n}^{2}$ test statistics are calculated by substituting the $z_{(i)}, i=1,2, \ldots r$ values and the nonparametric estimator $\hat{F}(x)$ in place of the EDF in equations (3-3) and (3-4).

$\underline{\text { Step } 8}$ Steps 1-7 are repeated 10000 times to generate 10000 independent test statistics for the Cramer von Mises as well as Anderson- Darling tests.

$\underline{\text { Step } 9}$ For each type, the 10000 test statistic are ordered, and the $80^{\text {th }}, 85^{\text {th }}, 90^{\text {th }}, 95^{\text {th }}$ and $99^{\text {th }}$ percentiles are found. These percentiles approximate the critical values for respective significance levels $\alpha$ of $0.20,0.15,0.10,0.05,0.01$.

\section{POWER COMPARISON AND RESULTS}

The corresponding power study for testing the hypotheses is conducted under $H_{0}$ and the power is computed. The test shows powers, which were reasonably close to the $\alpha$ levels.

The power of a statistical test is the probability of correctly rejecting a false null hypothesis. In our case, the null hypothesis $\left(H_{0}\right)$ is that the censored sample $x_{(1)}<x_{(2)}<\ldots<x_{(r)}$ comes from the normal distribution. The alternative hypothesis $\left(H_{a}\right)$ is that the sample follows some other distribution. The following alternative distributions are considered:

- $H_{1}$ : Uniform over the range 0.0 to 1.0 .

- $H_{2}$ : Chi square with 1 degree of freedom.

- $H_{3}$ : Chi square with 4 degree of freedom.

- $H_{4}$ : Negative Exponential.

- $H_{5}$ : Cauchy.

- $H_{6}$ : Double Exponential.

- $H_{7}$ : t-student distribution with 3 d.f.

- $H_{8}$ : Logistic distribution.

- $H_{9}$ : Normal distribution. 
These distributions cover a wide spectrum of various shapes. Some of these shapes are close to the original distribution. However some of them are dramatically far a way from the normal shape such as uniform, Chi square with 1 and 4 degrees of freedom and Exponential distributions, such distributions are considered to show how good the proposed test can discriminates any censored sample that comes from these distributions.

The sample size $n$ is varied from 10 to 60 with increments of 10 observations, and the significance levels $\alpha$, again include $0.20,0.15,0.10,0.05$ and 0.01 , for each alternative.

The following steps describe the procedure for establishing tests under the predefined nine alternatives.

$\underline{\text { Step } 1}$ A sample of $\mathrm{n}$ random variates is generated from the selected alternative distributions.

Step 2 The null hypothesis $H_{0}$ is assumed and steps 2-7 of the critical value generation procedure are performed to compute values for the $\mathrm{CvM}$ and $\mathrm{AD}$ test statistics.

$\underline{\text { Step } 3}$ For the given distribution and significance level $\alpha, H_{0}$ is rejected if the test statistic exceeds the corresponding critical value.

Step 4 Steps 1-3 are repeated 10000 times to generate 10000 independent sets of test statistic values.

Step 5 The power of each test is obtained by counting the number of times in which $H_{0}$ is rejected divided by 10000 .

The results for the proposed goodness of fit test for censored normal samples are shown in Table lup to Table 16. Table 1 gives the critical values at different significant levels in case of the modified CvM test statistic. Table 2 shows the power of the test in case of censored normal samples different from those used to determine the critical values in Table 1. The power of the test in this table is close enough to the corresponding $\alpha$-levels which means that the test performed right with normal distribution. Table 3 to Table 8 show the performance of the test under the nine different alternatives. These tables show an increasing power when the sample size increase. The test discriminates all alternatives with high power except for the logistic distribution which has a shape close to the normal.

Similar tables (Table 9 - Table 16) are describing results in case of AD test. Finally it is noted that the modified test using the AD statistic gives better power than the test based on the CvM statistic for different alternatives. 
Table 1. Simulated Critical Values for the New Suggested Test for Normal Censored samples at Censored ratio 0.6 with Sample Sizes n = 10 (10) 60 (Using CvM) (at

Significance Levels $\alpha=.2, .15, .1, .05, .01)$

\begin{tabular}{|c||c||c||c|c|c|}
\hline $\mathrm{N}$ & 0.20 & 0.15 & 0.10 & 0.05 & .01 \\
\hline \hline 10 & 0.0285 & 0.0328 & 0.0392 & 0.0536 & 0.0958 \\
20 & 0.0326 & 0.0375 & 0.0447 & 0.0601 & 0.1201 \\
30 & 0.0337 & 0.0385 & 0.0458 & 0.0603 & 0.1080 \\
40 & 0.0341 & 0.0390 & 0.0461 & 0.0606 & 0.0984 \\
50 & 0.0342 & 0.0389 & 0.0469 & 0.0606 & 0.1054 \\
60 & 0.0345 & 0.0392 & 0.0463 & 0.0597 & 0.1006 \\
\hline
\end{tabular}

Table 2. Power of Tests for the Normal Censored samples at Censored ratio 0.6 for Sample Sizes n $=10$ (10) 60 (Using CvM) (at Significance Levels $\alpha=.2, .15, .1, .05, .01$ )

\begin{tabular}{|c|c||c|c|c|c|}
\hline $\mathrm{N}$ & 0.20 & 0.15 & 0.10 & 0.05 & .01 \\
\hline \hline 10 & 0.2096 & 0.1533 & 0.1064 & 0.0534 & 0.0139 \\
20 & 0.1980 & 0.1490 & 0.0998 & 0.0499 & 0.0080 \\
30 & 0.1996 & 0.1527 & 0.1044 & 0.0535 & 0.0096 \\
40 & 0.1980 & 0.1487 & 0.0994 & 0.0503 & 0.0109 \\
50 & 0.2084 & 0.1565 & 0.0989 & 0.0491 & 0.0101 \\
60 & 0.2030 & 0.1522 & 0.1015 & 0.0517 & 0.0099 \\
\hline
\end{tabular}

Table 3. Power of the test for Normal censored sample of size 10 at censored ratio 6 (Using CvM)(Normal against one of the following:)

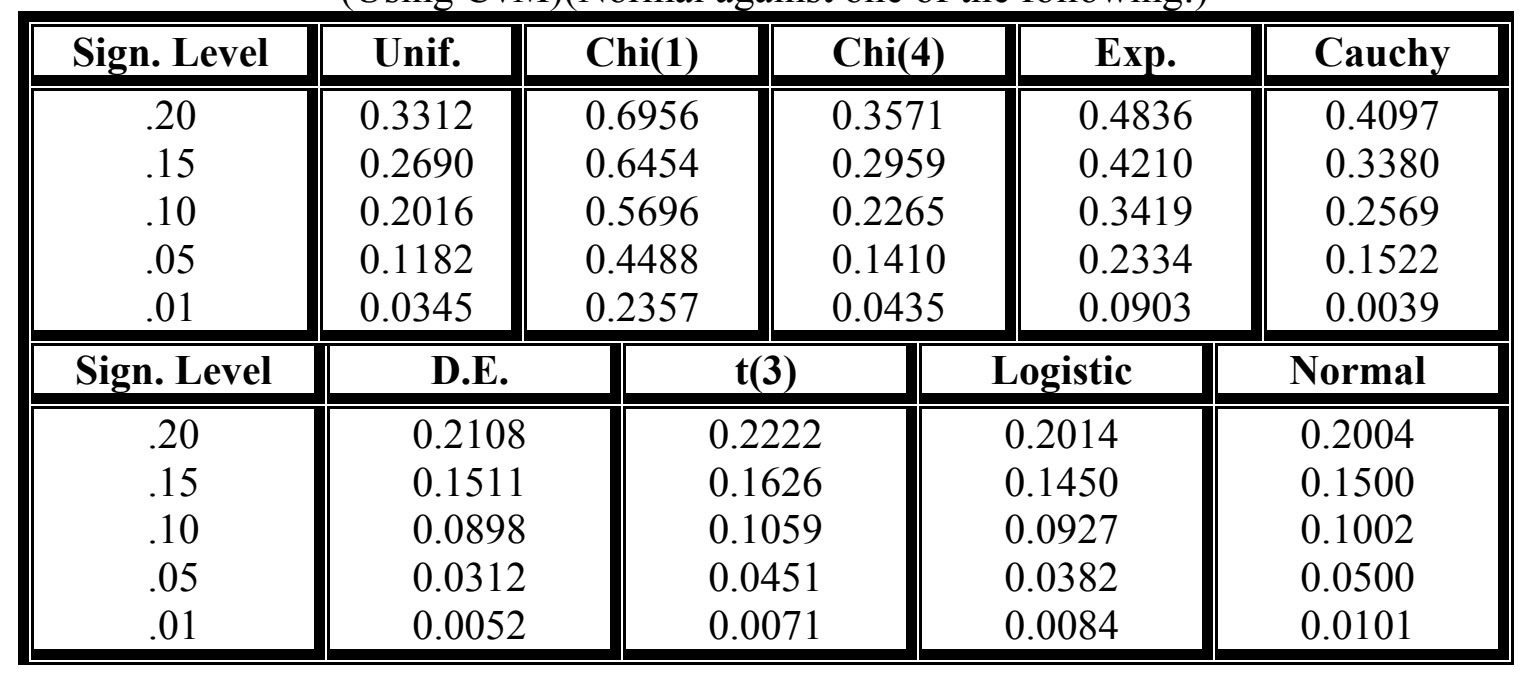


Table 4. Power of the test for Normal censored sample of size 20 at censored ratio 6 (Using CvM) (Normal against one of the following:)

\begin{tabular}{|c|c|c|c|c|c|}
\hline Sign.Lelvel & Unif. & $\overline{~ C h i(1) ~}$ & $\overline{C \text { Chi(4) }}$ & Exp. & Cauchy \\
\hline $\begin{array}{l}.20 \\
.15 \\
.10 \\
.05 \\
.01\end{array}$ & $\begin{array}{l}0.4821 \\
0.4137 \\
0.3359 \\
0.2326 \\
0.0698 \\
\end{array}$ & $\begin{array}{l}0.9377 \\
0.9174 \\
0.8879 \\
0.8208 \\
0.5876 \\
\end{array}$ & $\begin{array}{l}0.4986 \\
0.4387 \\
0.3628 \\
0.2539 \\
0.0804\end{array}$ & $\begin{array}{l}0.7321 \\
0.6775 \\
0.6075 \\
0.4875 \\
0.2174 \\
\end{array}$ & $\begin{array}{l}0.6791 \\
0.6228 \\
0.5550 \\
0.4302 \\
0.1518 \\
\end{array}$ \\
\hline Sign. Level & D.E. & & & Logistic & Normal \\
\hline $\begin{array}{l}.20 \\
.15 \\
.10 \\
.05 \\
.01\end{array}$ & $\begin{array}{l}0.2559 \\
0.1868 \\
0.1219 \\
0.0480 \\
0.0015 \\
\end{array}$ & & & $\begin{array}{l}0.1997 \\
0.1465 \\
0.0928 \\
0.0410 \\
0.0046 \\
\end{array}$ & $\begin{array}{l}0.2001 \\
0.1501 \\
0.1002 \\
0.0501 \\
0.0100\end{array}$ \\
\hline
\end{tabular}

Table 5. Power of the test for Normal censored sample of size 30 at censored ratio. 6 (Using CvM) (Normal against one of the following:)

\begin{tabular}{|c|c|c|c|c|c|}
\hline Sign. Level & Unif. & Chi(1) & Chi(4) & Exp. & Cauchy \\
\hline $\begin{array}{l}.20 \\
.15 \\
.10 \\
.05 \\
.01\end{array}$ & $\begin{array}{l}0.6189 \\
0.5579 \\
0.4777 \\
0.3561 \\
0.1527 \\
\end{array}$ & $\begin{array}{l}0.9895 \\
0.9865 \\
0.9795 \\
0.9595 \\
0.8683\end{array}$ & $\begin{array}{l}0.6273 \\
0.5707 \\
0.4955 \\
0.3741 \\
0.1687\end{array}$ & $\begin{array}{l}0.8779 \\
0.8459 \\
0.8008 \\
0.7113 \\
0.4590 \\
\end{array}$ & $\begin{array}{l}0.8284 \\
0.7972 \\
0.7508 \\
0.6592 \\
0.4047 \\
\end{array}$ \\
\hline Sign. Level & D.E. & & & stic & Normal \\
\hline $\begin{array}{l}.20 \\
.15 \\
.10 \\
.05 \\
.01\end{array}$ & $\begin{array}{l}0.3285 \\
0.2609 \\
0.1800 \\
0.0843 \\
0.0052\end{array}$ & & & & $\begin{array}{l}0.2001 \\
0.1499 \\
0.1002 \\
0.0501 \\
0.0100\end{array}$ \\
\hline
\end{tabular}

Unif. $=$ Uniform $\quad$ Chi $(\mathrm{k})=$ Chi square with $\mathrm{k}$ d.f $\quad$ Exp. $=$ Negative Exponential D.E. $=$ Double Exponential $\mathrm{t}(3)=\mathrm{t}$-student distribution with 3 d.f.

Table 6. Power of the test for Normal censored sample of size 40 at censored ratio 6 (Using CvM) (Normal against one of the following:)

\begin{tabular}{|c||c||c||c||c||c|}
\hline Sign. Level & Unif. & Chi(1) & Chi(4) & Exp. & Cauchy \\
\hline \hline .20 & 0.7341 & 0.9988 & 0.7286 & 0.9441 & 0.9197 \\
.15 & 0.6794 & 0.9982 & 0.6760 & 0.9246 & 0.9020 \\
.10 & 0.6076 & 0.9971 & 0.6011 & 0.8963 & 0.8743 \\
.05 & 0.4742 & 0.9918 & 0.4805 & 0.8360 & 0.8141 \\
.01 & 0.2564 & 0.9667 & 0.2754 & 0.6650 & 0.6338 \\
\hline
\end{tabular}




\begin{tabular}{|c|c|c|c|c|}
\hline Sign. Level & $\overline{\text { D.E. }}$ & $t(3)$ & Logistic & Normal \\
\hline $\begin{array}{l}.20 \\
.15 \\
.10 \\
.05 \\
.01\end{array}$ & $\begin{array}{l}0.4130 \\
0.3351 \\
0.2508 \\
0.1317 \\
0.0184\end{array}$ & $\begin{array}{l}0.4460 \\
0.3831 \\
0.3109 \\
0.2005 \\
0.0693\end{array}$ & $\begin{array}{l}0.2276 \\
0.1720 \\
0.1144 \\
0.0503 \\
0.0081\end{array}$ & $\begin{array}{l}0.2001 \\
0.1501 \\
0.1002 \\
0.0502 \\
0.0101\end{array}$ \\
\hline
\end{tabular}

Table 7. Power of the test for Normal censored sample of size 50 at censored ratio 6 (Using CvM) (Normal against one of the following:)

\begin{tabular}{||c||c|c||c|c|c|}
\hline Sign. Level & Unif. & Chi(1) & Chi(4) & Exp. & Cauchy \\
\hline \hline .20 & 0.8183 & 0.9999 & 0.7993 & 0.9787 & 0.9642 \\
.15 & 0.7760 & 0.9999 & 0.7579 & 0.9692 & 0.9554 \\
.10 & 0.7036 & 0.9998 & 0.6943 & 0.9527 & 0.9353 \\
.05 & 0.5812 & 0.9992 & 0.5876 & 0.9169 & 0.8979 \\
.01 & 0.3110 & 0.9901 & 0.3289 & 0.7640 & 0.7467 \\
\hline & & & & & \\
\hline Sign. Level & D.E. & \multicolumn{2}{|c|}{$\mathrm{t}(3)$} & Logistic & Normal \\
\hline \hline .20 & 0.4922 & 0.5163 & \multicolumn{2}{|c|}{0.2504} & 0.2001 \\
.15 & 0.4235 & 0.4566 & 0.1934 & 0.1500 \\
.10 & 0.3148 & 0.3733 & 0.1234 & 0.1002 \\
.05 & 0.1887 & 0.2571 & 0.0575 & 0.0502 \\
.01 & 0.0258 & 0.0864 & 0.0052 & 0.0100 \\
\hline
\end{tabular}

Table 8. Power of the test for Normal censored sample of size 60 at censored ratio 6 (Using CvM) (Normal against one of the following:)

\begin{tabular}{|c||c|c|c||c|c||}
\hline Sign. Level & Unif. & Chi(1) & Chi(4) & Exp. & Cauchy \\
\hline \hline .20 & 0.8799 & 1.0000 & 0.8575 & 0.9924 & 0.9801 \\
.15 & 0.8487 & 1.0000 & 0.8263 & 0.9889 & 0.9751 \\
.10 & 0.7944 & 1.0000 & 0.7749 & 0.9804 & 0.9649 \\
.05 & 0.6893 & 0.9998 & 0.6813 & 0.9624 & 0.9422 \\
.01 & 0.4180 & 0.9981 & 0.4373 & 0.8701 & 0.8561 \\
\hline \hline Sign. Level & D.E. & \multicolumn{1}{|c|}{ t(3) } & \multicolumn{2}{|c||}{ Logistic } & Normal \\
\hline \hline .20 & 0.5606 & 0.5702 & \multicolumn{2}{|c|}{0.2686} & 0.1999 \\
.15 & 0.4893 & 0.5134 & & 0.2101 & 0.1501 \\
.10 & 0.3951 & 0.4387 & 0.1441 & 0.1001 \\
.05 & 0.2564 & 0.3241 & & 0.0707 & 0.0501 \\
.01 & 0.0557 & 0.1255 & 0.0079 & 0.0101 \\
\hline
\end{tabular}


Table 9. Simulated Critical Values for the New Suggested Test for Normal Censored

Samples at censored ratio 0.6 with Sample Sizes $n==10$ (10) 60 (Using AD) (at

Significance Levels $\alpha=.2, .15, .1, .05, .01)$

\begin{tabular}{|c||c||c||c|c|c|}
\hline $\mathrm{N}$ & 0.20 & 0.15 & 0.10 & 0.05 & .01 \\
\hline \hline 10 & 0.1723 & 0.1959 & 0.2290 & 0.2970 & 0.5258 \\
20 & 0.1975 & 0.2256 & 0.2648 & 0.3410 & 0.6525 \\
30 & 0.2051 & 0.2308 & 0.2705 & 0.3469 & 0.5667 \\
40 & 0.2082 & 0.2332 & 0.2719 & 0.3424 & 0.5325 \\
50 & 0.2083 & 0.2352 & 0.2730 & 0.3472 & 0.5501 \\
60 & 0.2104 & 0.2367 & 0.2719 & 0.3388 & 0.5373 \\
\hline
\end{tabular}

Table 10. Power of Tests for Normal Censored Samples at Censored ratio 0.6 for Sample Sizes $n=10$ (10) 60 (Using AD) (at Significance Levels $\alpha=.2, .15, .1, .05, .01$ )

\begin{tabular}{|c||c|c|c|c|c|}
\hline $\mathbf{N}$ & $\mathbf{0 . 2 0}$ & $\mathbf{0 . 1 5}$ & $\mathbf{0 . 1 0}$ & $\mathbf{0 . 0 5}$ & $\mathbf{. 0 1}$ \\
\hline \hline 10 & 0.2096 & 0.1550 & 0.1067 & 0.0546 & 0.0135 \\
20 & 0.2016 & 0.1466 & 0.0989 & 0.0507 & 0.0079 \\
30 & 0.1990 & 0.1531 & 0.1015 & 0.0528 & 0.0107 \\
40 & 0.1982 & 0.1521 & 0.1003 & 0.0506 & 0.0107 \\
50 & 0.2080 & 0.1539 & 0.1040 & 0.0480 & 0.0111 \\
60 & 0.2019 & 0.1534 & 0.1046 & 0.0526 & 0.0100 \\
\hline
\end{tabular}

Table 11. Power of the test for Normal censored sample of size 10 at censored ratio .6 (Using AD) (Normal against one of the following:)

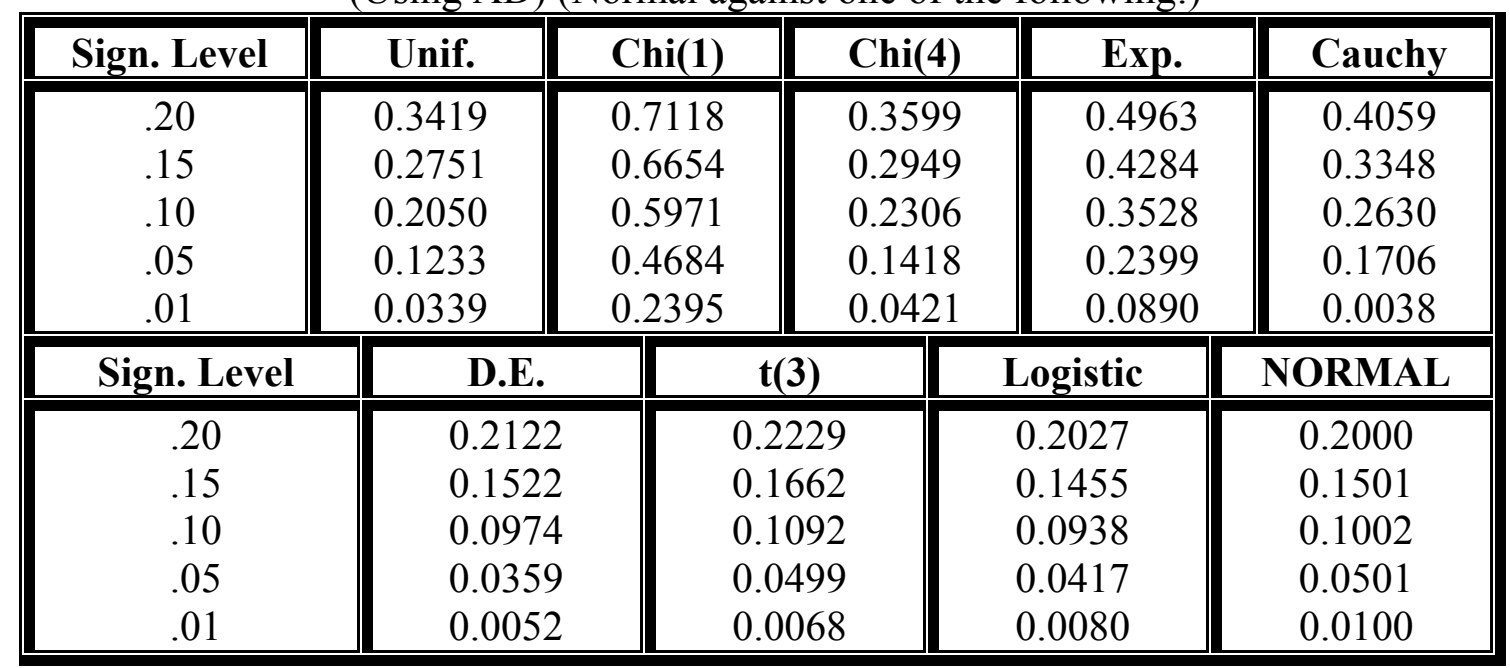


Table 12. Power of the test for Normal censored sample of size 20 at censored ratio .6 (Using AD) (Normal against one of the following:)

\begin{tabular}{|c|c|c|c|c|c|}
\hline Sign. Level & Unif. & Chi(1) & Chi(4) & Exp. & Cauchy \\
\hline $\begin{array}{l}.20 \\
.15 \\
.10 \\
.05 \\
.01 \\
\end{array}$ & $\begin{array}{l}0.4978 \\
0.4249 \\
0.3405 \\
0.2369 \\
0.0692 \\
\end{array}$ & $\begin{array}{l}0.9503 \\
0.9317 \\
0.9039 \\
0.8421 \\
0.6067 \\
\end{array}$ & $\begin{array}{l}0.4993 \\
0.4347 \\
0.3562 \\
0.2460 \\
0.0794 \\
\end{array}$ & $\begin{array}{l}0.7479 \\
0.6905 \\
0.6159 \\
0.4957 \\
0.2238 \\
\end{array}$ & $\begin{array}{l}0.6894 \\
0.6424 \\
0.5754 \\
0.4751 \\
0.2198 \\
\end{array}$ \\
\hline Sign. Level & D.E. & & & logistic & NORMAL \\
\hline $\begin{array}{l}.20 \\
.15 \\
.10 \\
.05 \\
.01\end{array}$ & $\begin{array}{l}0.2697 \\
0.2071 \\
0.1416 \\
0.0685 \\
0.0050\end{array}$ & & & $\begin{array}{l}0.2109 \\
0.1532 \\
0.0989 \\
0.0482 \\
0.0050 \\
\end{array}$ & $\begin{array}{l}0.2000 \\
0.1500 \\
0.1001 \\
0.0502 \\
0.0100 \\
\end{array}$ \\
\hline
\end{tabular}

Table 13. Power of the test for Normal censored sample of size 30 at censored ratio 6 (Using AD) (Normal against one of the following:)

\begin{tabular}{|c|c|c|c|c|c|}
\hline Sign. Level & Unif. & Chi(1) & Chi(4) & Exp. & Cauchy \\
\hline $\begin{array}{l}.20 \\
.15 \\
.10 \\
.05 \\
.01 \\
\end{array}$ & $\begin{array}{l}0.6465 \\
0.5807 \\
0.4933 \\
0.3686 \\
0.1675 \\
\end{array}$ & $\begin{array}{l}0.9932 \\
0.9906 \\
0.9850 \\
0.9690 \\
0.8966 \\
\end{array}$ & $\begin{array}{l}0.6281 \\
0.5689 \\
0.4894 \\
0.3709 \\
0.1751 \\
\end{array}$ & $\begin{array}{l}0.8908 \\
0.8599 \\
0.8143 \\
0.7239 \\
0.4899 \\
\end{array}$ & $\begin{array}{l}0.8377 \\
0.8098 \\
0.7702 \\
0.6917 \\
0.5040 \\
\end{array}$ \\
\hline Sign. Level & D.E. & & & logistic & NORMAL \\
\hline $\begin{array}{l}.20 \\
.15 \\
.10 \\
.05 \\
.01 \\
\end{array}$ & $\begin{array}{l}0.3487 \\
0.2866 \\
0.2091 \\
0.1155 \\
0.0195 \\
\end{array}$ & & & $\begin{array}{l}0.2228 \\
0.1729 \\
0.1149 \\
0.0557 \\
0.0092 \\
\end{array}$ & $\begin{array}{l}0.2000 \\
0.1500 \\
0.1002 \\
0.0501 \\
0.0100\end{array}$ \\
\hline
\end{tabular}


Table 14. Power of the test for Normal censored sample of size 40 at censored ratio .6 (Using AD) (Normal against one of the following:)

\begin{tabular}{|c||c|c|c|c|c|}
\hline Sign. Level & Unif. & Chi(1) & Chi(4) & Exp. & Cauchy \\
\hline .20 & 0.7659 & 0.9994 & 0.7366 & 0.9572 & 0.9253 \\
.15 & 0.7144 & 0.9992 & 0.6808 & 0.9405 & 0.9113 \\
.10 & 0.6404 & 0.9985 & 0.6021 & 0.9100 & 0.8867 \\
.05 & 0.5090 & 0.9956 & 0.4848 & 0.8565 & 0.8390 \\
.01 & 0.2734 & 0.9775 & 0.2748 & 0.6907 & 0.7037 \\
\hline Sign. Level & \multicolumn{2}{|c|}{ D.E. } & \multicolumn{2}{|c|}{ t(3) } & \multicolumn{2}{|c|}{ logistic } & NORMAL \\
\hline .20 & 0.4333 & 0.4769 & 0.2452 & 0.2000 \\
.15 & 0.3649 & 0.4233 & 0.1944 & 0.1500 \\
.10 & 0.2795 & 0.3516 & 0.1358 & 0.1001 \\
.05 & 0.1772 & 0.2561 & 0.0694 & 0.0501 \\
.01 & 0.0461 & 0.1191 & 0.0132 & 0.0101 \\
\hline
\end{tabular}

Table 15. Power of the test for Normal censored sample of size 50 at censored ratio 6 (Using AD) (Normal against one of the following:)

\begin{tabular}{|c|c|c|c|c|c|}
\hline Sign. Level & Unif. & Chi(1) & Chi(4) & Exp. & Cauchy \\
\hline .20 & 0.8512 & 1.0000 & 0.8096 & 0.9856 & 0.9679 \\
.15 & 0.8094 & 1.0000 & 0.7649 & 0.9765 & 0.9579 \\
.10 & 0.7489 & 0.9999 & 0.7032 & 0.9649 & 0.9438 \\
.05 & 0.6226 & 0.9997 & 0.5898 & 0.9311 & 0.9136 \\
.01 & 0.3568 & 0.9954 & 0.3489 & 0.8018 & 0.8145 \\
\hline Sign. Level & \multicolumn{2}{|c|}{ D.E. } & \multicolumn{2}{|c|}{ t(3) } & \multicolumn{2}{|c|}{ logistic } & NORMAL \\
\hline .20 & 0.5108 & 0.5498 & 0.2760 & 0.2000 \\
.15 & 0.4392 & 0.4917 & 0.2136 & 0.1501 \\
.10 & 0.3506 & 0.4222 & 0.1494 & 0.1001 \\
.05 & 0.2280 & 0.3162 & 0.0749 & 0.0501 \\
.01 & 0.0605 & 0.1471 & 0.0124 & 0.0100 \\
\hline
\end{tabular}


Table 16. Power of the test for Normal censored sample of size 60 at censored ratio .6 (Using AD) (Normal against one of the following:)

\begin{tabular}{|c|c|c|c|c|c|}
\hline Sign. Level & Unif. & Chi(1) & Chi(4) & Exp. & Cauchy \\
\hline $\begin{array}{l}.20 \\
.15 \\
.10 \\
.05 \\
.01 \\
\end{array}$ & $\begin{array}{l}0.9067 \\
0.8779 \\
0.8359 \\
0.7374 \\
0.4727 \\
\end{array}$ & $\begin{array}{l}1.0000 \\
1.0000 \\
1.0000 \\
1.0000 \\
0.9991 \\
\end{array}$ & $\begin{array}{l}0.8690 \\
0.8321 \\
0.7854 \\
0.6906 \\
0.4475 \\
\end{array}$ & $\begin{array}{l}0.9946 \\
0.9924 \\
0.9874 \\
0.9731 \\
0.8970 \\
\end{array}$ & $\begin{array}{l}0.9810 \\
0.9768 \\
0.9691 \\
0.9516 \\
0.8886 \\
\end{array}$ \\
\hline Sign. Level & D.E. & & & istic & NORMAL \\
\hline $\begin{array}{l}.20 \\
.15 \\
.10 \\
.05 \\
.01\end{array}$ & $\begin{array}{l}0.5737 \\
0.5052 \\
0.4220 \\
0.3003 \\
0.0963\end{array}$ & & & $\begin{array}{l}925 \\
316 \\
700 \\
955 \\
169 \\
\end{array}$ & $\begin{array}{l}0.2000 \\
0.1500 \\
0.1002 \\
0.0503 \\
0.0101 \\
\end{array}$ \\
\hline
\end{tabular}

\section{REFERENCES}

1. Ali, M. M. and chan, L. K., On Gupta's estimates of the parameters of the normal distribution. Biometrica, 51, 498-501, 1964

2. Blom, G., Statistical Estimates and Transformed Beta Variates. New York: Wiley, 1958.

3. Choulakian, V., Lockhart R.A. and Stephens, M.A. Cramer von Mises statistics for discrete distributions, the Canadian Journal of statistics, 22, 125 - 137, 1994.

4. D’Agostino, R. B. and Stephens, M. A., Goodness of fit techniques. Marcel

Dekker, Inc. New York and Basel, 1986.

5. Gunes, H., Dietz, D. C., Auclair, P. F.and Moore, A. H., Modified goodness - of fit tests for the inverse Gaussian distribution. Computational statistics \&_Data analysis, $24,63-77,1997$.

6. Gupta, A. K., Estimation of the mean and standard deviation of a normal population from a censored data. Biometrika, 67, 133-143, 1952.

7. Lilliefors, H. W., On the Kolmogorov test for normality with mean and variance unknown JASA, 62, $143-147,1967$.

8. Marks, N. B., Modification of the Kolmogrov Smirnov test for the Erlang-2 Distribution. Commun. Statist. Simula, $27,39-49,1998$.

9. Pettitt, A. N. and Stephens, M.A., Modified Cramer-von Mises statistics for censored data. Biometrika, 63, 291-298, 1976.

10. Silverman, B. W. , Density estimation for statistics and data analysis. Chapman and Hall Ltd. 1986.

11. Woodruff, B. W. and A. H. Moore et al. , Modified goodness of fit tests for gamma distribution with unknown location and scale parameters. IEEE transactions on Reliability, 33, $241-245,1984$.

12. Woodruff, B. W. and A. H. Moore et al., Modified goodness of fit tests for logistic distribution with unknown location and scale parameters. Stat. Simula Comp, 15(1), 77 $-83,1986$. 